\title{
Daubechies12 Wavelet in Analyzing the Current of Anode Rod
}

\author{
Gaoqi Xiao \\ School of Automation and Electrical Engineering \\ University of Science and Technology Beijing \\ Beijing, China \\ xiaogaoqichn@hotmail.com
}

\begin{abstract}
This paper is concerned about the rod current of aluminum reduction cell. Daubechies12 wavelet was adapted to analyze time-frequency characteristics of anode current collected which was carried on the pretreatment, and the experimental results verified the effectiveness of the proposed scheme, compared with actual working conditions. After using Daubechies12 wavelet, we can get the magnetic fluid fluctuation frequency. In this way, we obtain the key factors of fault diagnosis. This research can make preparations for the follow-up study of the fault diagnosis of aluminum electrolysis.
\end{abstract}

Keywords-Aluminum electrolysis; Anode current; Data Collection; Mallat algorithm; Daubechies12 wavelet transform

\section{INTRODUCTION}

What we study in this paper about the data analysis is essentially the process of the current signal.Signal processing, as a science, is more and more important in science and engineering technology.Wavelet analysis is achievement of functional analysis 、 Fourier analysis 、 spline analysis 、 harmonic analysis and numerical analysis. Wavelet analysis is a method of analyzing the time-dimension of a signal. It has a character of multi-resolution analysis and also has the ability to detail local characters of a signal in both time and frequency. Its size of transforming window keeps constantly, but the shape of both its time window andfrequency window may be changeable.The spectrogram obtained from the wavelet transform has good frequency resolution in low frequency and good time resolution in high frequency, this conditionhas more accord with the characters of anode current. It's quite fit for detecting normal signal which carries transient abnormal phenomena and showing its components. Therefore, we combine FFT transform and multi-resolution analysis of wavelet analysis theory to analysis the rod current.

\section{DyADIC WAVELET TRANSFORM AND MULTI-RESOLUTION ANALYSIS}

The basic idea of wavelet transform is to express and approximate a signal or function with a setof functions. These functionsare called the wavelets, which is composed of different scales of shifting transformation and dilating transformationon the basic wavelet function. The most important feature of wavelets is that theproduct of time and frequencyis small. Focusing on the time axis and frequency axis, it can be very good to bring out the local characteristics of the signal [1]. Wavelet function is defined as follows,

Our common functions are generally $L^{2}(R) \bigcap L^{1}(R)$, Suppose $\psi(t) \in L^{2}(R) \cap L^{1}(R)$, and satisfy the condition

$$
C_{\psi}=\int_{-\infty}^{+\infty} \frac{|\hat{\psi}(\omega)|^{2}}{|\omega|} d \omega<+\infty(1)
$$

then $\psi(t)$ is a basic wavelet function. (1) is called admissible condition. From this condition, it is necessary to have $\hat{\psi}(0)=0$, namely $\int_{-\infty}^{+\infty} \psi(t) d t<+\infty$, wavelet basis function at least has first order vanishing moment.

\section{A. Dyadic Wavelet Transform}

For the wavelet sequence whose scale and shift are discrete. If you take the discrete grid $a_{0}=2, b_{0}=0$, it is equivalent to the continuous wavelet of the binary discrete only in the scale, and the displacement is still continuous variation, and this type of wavelet is called dyadic wavelet,

$$
\psi_{j b}(t)=2^{-j / 2} \psi\left(2^{-j} t-b\right)(2)
$$

Only when there exists A and B which are constants,

$0<\mathrm{A} \leq B<\infty$, and that

$$
\mathrm{A} \leq \sum_{j \in z}\left|\psi\left(2^{j} \omega\right)\right|^{2} \leq B^{(3)}
$$

can the definition of dyadic wavelet be practical. Formula(3) is called stability condition of dyadic wavelet, when $A=B$, it is called the most stability condition. The definition of dyadic wavelet transform is

$$
W T_{2^{j}}(b)=2^{-j / 2} \sum_{j \in Z} \int_{R} W T_{2^{j}}(b) \psi\left(\frac{b-t}{2^{j}}\right) d t(4)
$$

The reconstruction formula of dyadic wavelet is 


$$
f(t)=2^{-j / 2} \sum_{j \in Z} \int_{R} W T_{2^{j}}(b) \psi\left(\frac{b-t}{2^{j}}\right) d t(5)
$$

Dyadic wavelet is a type of discrete wavelet which is different from continuous wavelet, it is only discretized in the scale parameter, and the shift parameter is still continuous variation in time domain. Although the dyadic wavelet transform has a large redundancy, it preserves the invariant property of the signal in time domain.

\section{B. Multi-resolution Analysis AND Mallat algorithm}

The basic idea of multi-resolution analysis is firstly to establish basement in the subspace of $L^{2}(R)$, and then use dilation and translation transform to handle this subspace to the $L^{2}(R)$.

$\left\{V_{j}\right\}_{j \in Z}$ is a closed subspace of $L^{2}(R),\left\{V_{j}\right\}_{j \in Z}$ is called a multi-resolution analysis of $L^{2}(R)$, if $\left\{V_{j}\right\}_{j \in Z}$ meet the following four conditions:

1) Uniform monotonic, $V_{j} \subset V_{j+1}, j \in Z$

2) Asymptotic completeness, $\bigcap_{j \in Z} V_{j}=\{0\}, \overline{j \in Z} V_{j}=L^{2}(R)$

3) Expansion rule, $\quad f(t) \in V_{j} \Leftrightarrow f(2 t) \in V_{j+1}, j \in Z$

4) Existence of orthogonal basis, let $V_{0}$ be the standard orthogonal basis of $\varphi(t) \in V_{0}$ such that $\{\varphi(t-k)\}_{k \in Z}$ exists. $\varphi$ is called scaling function, $V_{0}$ is called approximation space.

Multi-resolution analysis $\left\{V_{j}\right\}_{j \in Z}$ satisfying the above conditions is also known as the multi-resolution analysis which is generated by $\varphi$.

If a series of closed subspace $\left\{V_{j}\right\}_{j \in Z}$ of function space $L^{2}(R)$ is a multi-resolution analysis (or approximation) of $L^{2}(R) \quad, \quad \varphi(t) \in L^{2}(R)$ will be a scale function, $\varphi_{j, k}(t)=2^{j / 2} \varphi\left(2^{j} t-k\right)$ can be the orthogonal complement space of scale space $j$, namely $V_{j+1}=V_{j} \oplus W_{j}$, wavelet function $\psi_{j, k}(t)=2^{j / 2} \psi\left(2^{j} t-k\right)$ is orthonormal base of $W_{j}$. The subspace can be decomposed into

$$
V_{J}=V_{J-1} \oplus W_{J-1}=V_{J-2} \oplus W_{J-2} \oplus W_{J-1}=\ldots
$$

To any signal,

$$
x(t)=\sum_{k} c_{0}(k) \varphi(t-k)+\sum_{k} \sum_{j=0}^{J-1} d_{j}(k) 2^{j / 2} \psi\left(2^{j / 2}-k\right)(7)
$$

$\mathrm{J}$ means the number of layers of decomposition, $c_{0}(k)$ means scale coefficient, and $d_{j}(k)$ means wavelet coefficients.

If the scale function is an orthonormal basis, $c_{j}(k)$ and $d_{j}(k)$ can be expressed as,

$$
\left\{\begin{array}{l}
c_{j}(k)=<x(t), \varphi_{j, k}(t)> \\
d_{j}(k)=<x(t), \psi_{j, k}(t)>
\end{array}\right.
$$

From the two scale equations, we know,

$$
\left\{\begin{array}{l}
c_{j-1}(k)=\sum_{R} h(n-2 k) c_{j}(n) \\
d_{j-1}(k)=\sum_{R} g(n-2 k) c_{j}(n)
\end{array}\right.
$$

$c_{j}(k)$ means the scale coefficient in the layer $\mathrm{j}, h(k)$ means low pass filter coefficient, and $g(k)$ means high pass filter coefficients.

And satisfy the condition that,

$$
g(k)=(-1)^{k} h(1-k)
$$

(10) is the famous Mallat Decomposition Algorithm.

The initial scale factor $C_{j}(k)$ can be sampled by the signal $x(t)$. If the signal sampling frequency is greater than the Nyquist frequency, then $c_{j}(k)$ can approach $x(t)$ very well. That is to say, we don't need the wavelet coefficients $d_{j}(k)$ under this condition.

From the above analysis and deduction above on, we get the wavelet coefficients of each frequency component from the signal decomposition, which can be expressed as the form of matrix:

$$
C_{\text {signal }}=\left[c_{0}, d_{0}, d_{1}, \ldots d_{J-1}\right]
$$

Consider the opposite process, i.e., the reconstruction process.The definition of multi resolution analysis can be described,

$$
c_{j}(n)=\sum_{k \in Z} h(n-2 k) c_{j-1}(k)+\sum_{k \in Z} g(n-2 k) d_{j-1}(k)
$$

(12) is the well-known Mallat Reconstruction Algorithm.

In actual calculation, $h(k)$ which is non-zero is limited, supposing $h(0), h(1), \cdots h(M-1)$ are all non-zero, other 
$h(k)$ are zero. By (10) we can know that $g(2-M), g(3-M), \cdots g(0), g(1)$ are also non zero and other $g(k)$ are zero [2]. Following is the Mallat algorithm,

Decomposition formula:

$$
\left\{\begin{array}{l}
c_{j-1}(k)=\sum_{n=2 k}^{2 k+M-1} h(n-2 k) c_{j}(n) \\
d_{j-1}(k)=\sum_{2 k+2-M}^{2 k+1} g(n-2 k) c_{j}(n)
\end{array}\right.
$$

If $C_{j}=\left\{c_{j k}\right\}_{k \in Z}$, schematic diagram of reconstruction algorithm is shown in Fig.1,

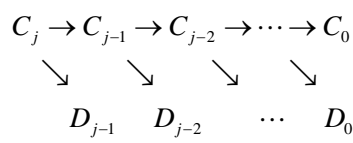

Fig. 1. Decomposition algorithm.

Reconstruction formula:

$c_{j}(n)=\sum_{k=[(n-M+1) / 2\rceil}^{\lfloor n / 2\rfloor} h(n-2 k) c_{j-1}(k)+\sum_{k=\lceil n / 2\rceil}^{\lfloor(n+M-1) / 2\rfloor} g(n-2 k) d_{j-1}(k)$

$\lfloor x\rfloor$ is the maximum integer less than $x$, and $\lceil x\rceil$ is the smallest integer not less than $X$.

\section{DETERMINATION OF DB12 WAVELET FUNCTION AND SELECTION OF DECOMPOSITION LEVEL}

Not only the industrial process of the multi variable coupling, time varying and large lag of the aluminum reduction cell, but also the complex physical and chemical reactions and the interference of various external conditions and operations lead the current signals to non-stationary signals. So we must choose the wavelet base function which is more sensitive to the irregular and has good locality both in time domain and frequency domain. In order to analyze the characteristics of the current signal in working condition, the wavelet analysis can extract the instantaneous and the abrupt change of the signal.

Therefore, the main consideration in the time domain and frequency domain is compactness characteristics when selecting the wavelet function. Daubechies wavelet has orthonormal character, time-frequency compactly supported character, high regularity character and can realized by Mallat quick arithmetic, so it is very suitable for the singularity of the signal detection[3]. This paper selects DB12 wavelet as the wavelet functions of the Mallat decomposition and reconstruction algorithm.

\section{A. Selection of decomposition level}

In order to determine the reasonable decomposition level, it is necessary to divide the frequency band of the signal, and the principle of frequency division is that the fundamental frequency of the signal is located at the center of the lowest sub frequency band. If the sampling frequency is $f_{s}$, then the level is obtained by (15) which is integralized[4].

$$
p=\log _{2}\left(\frac{f_{s}}{f_{0}} \sqrt{\frac{1}{8}}\right)+0.5
$$

$p$ means the layers of decomposition,$f_{s}$ means sampling frequency, and $f_{0}$ means the fundamental frequency.

In this paper, $f_{s}=10 \mathrm{~Hz}$. It is necessary to make further study about fundamental frequency, and the decomposition level depends on the specific circumstances.

\section{DATA ANALYSIS AND PROCESSING}

In an aluminum factory, the aluminum reduction cells of $200 \mathrm{kA}$ series adopt cryolite electrolyte for the subject of the molten state as solvent, alumina as solute, carbon as anode, aluminum liquid as the cathode, inlet 200kA DC power which has been rectified, and chemical reaction occurs in the poles. The aluminum reduction cells are in series. The 200KA is divided into 28 anode guide rod, and the current of each rod is different. The current of each pole also varies at the time axis.

In Fig. 2, the time domain of current curve is drawn by an anode rod, which can clearly see that the current is varied over time in the process of electrolysis.

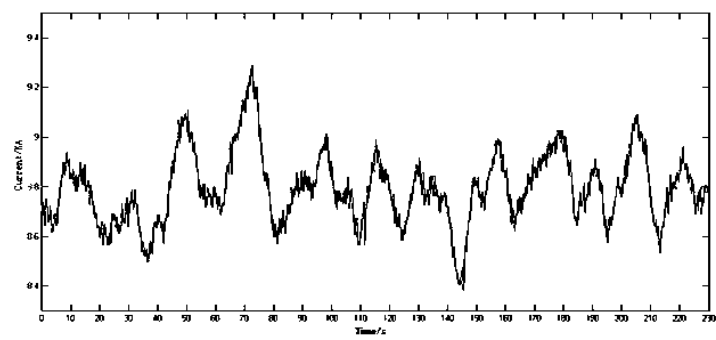

Fig. 2. Current fluctuation curve of anode rod B1.

The source of current fluctuation in aluminum reduction cell is molten electrolyte, which is the fluctuation of liquid aluminum(magnetic fluid). As the fluctuation of aluminum liquid, the electrolyte also fluctuates. The resistivity of the electrolyte is $0.5 \Omega \cdot \mathrm{cm}$, and the resistivity of aluminum liquid is $63 \times 10^{-5} \Omega \cdot \mathrm{cm}$. Therefore, the resistivity of the electrolyte 
is much higher than the resistivity of liquid aluminum and the current fluctuations is determined by the fluctuation of electrolyte layer [5-6].

In Fig.3, the sampling frequency is $10 \mathrm{~Hz}$ when using the DB12 wavelet function to carry on 9 levels of decomposition. According to the Shannon sampling theorem, the signal frequency is from 0 to $3 \mathrm{~Hz}$. The scale changes from $1 \mathrm{~Hz}$ to $10 \mathrm{~Hz}$, and the entire frequency band is divided into 9, after decomposition, we also can get $c_{0, r}(k)$ and $d_{j, r}(k)(0 \leq j \leq 10)$, there are 9 wavelet decomposition coefficients[7-8]. The high frequency components of the second, fourth, sixthand ninth layers are shown in Fig. 3.
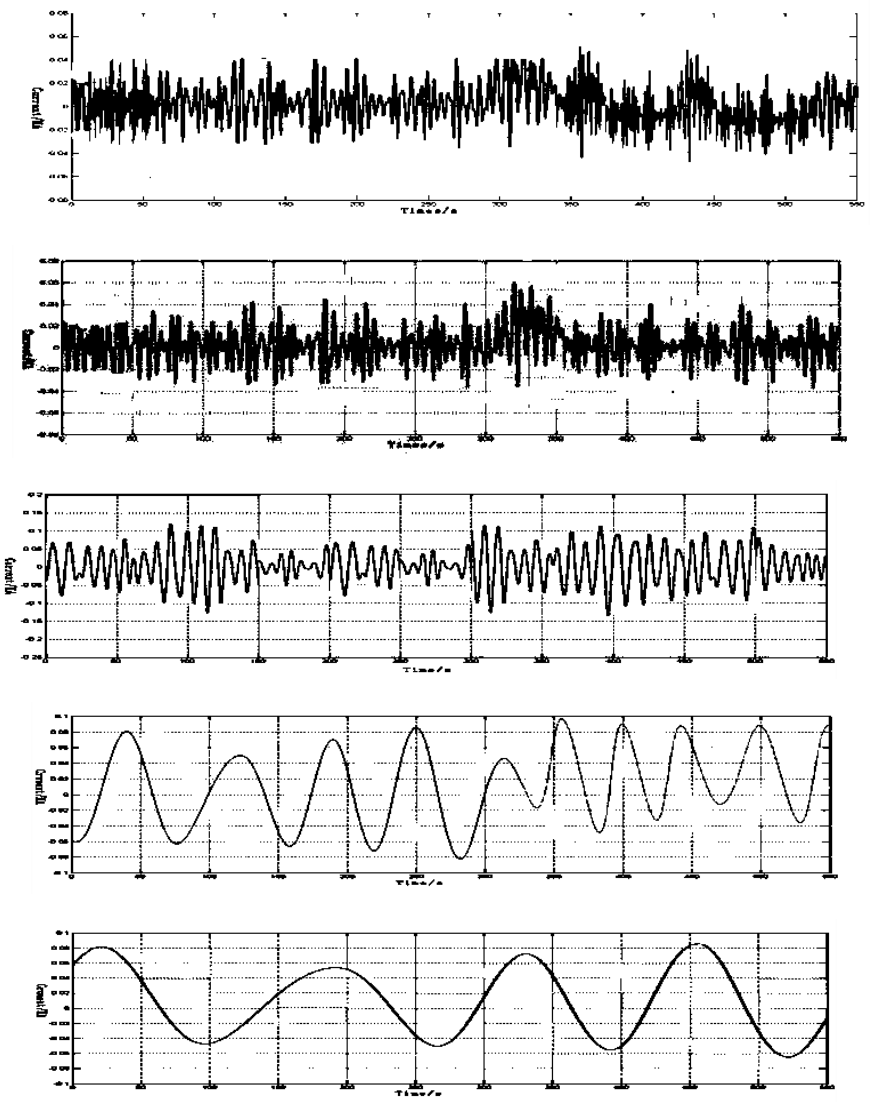

Fig. 3. Current fluctuation signal decomposition into 9 layer by DB12 wavelet of anode rod A1

After decomposition into 9 layers by DB12 wavelet function, it can be seen that the fundamental wave is a sine wave, and the tenth layer of the signal sequence is located between $0.005859 \mathrm{~Hz} 0.01171 \mathrm{~Hz}$. Removing DC component and noise in the signal, we can get Fourier spectrum analysis by using Fourier Transform shown in Fig.4, and the dominant frequency was located at the position of $0.0065 \mathrm{~Hz}$. In this way, we obtain the key factors of fault diagnosis.

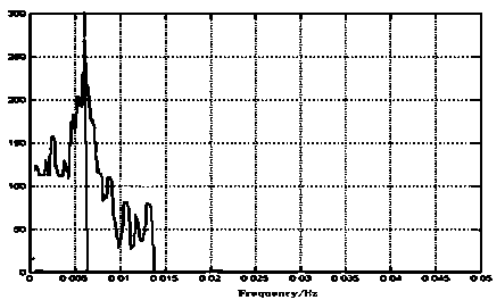

Fig. 4. Oscillation frequency of magnetic fluid

During the productionof aluminum electrolysis, the electromagnetic fluid fluctuation will affect the voltage of cells, and the frequency will destroy the normal operation of thecells, which we can judge the faults of the aluminum electrolysis, such as anode effect.

\section{CONCLUSIONS}

This paperanalysis a lot of data collected from the aluminum factory. After analyzing these data,we can gain some conclusions about current as follows,in normal condition, the DC statepresented in the current. However, the current slightly fluctuates from 9.7KA to 10.3KA under the effectmagnetic of fluid. The fluctuation frequency of the magnetic fluid is $0.0065 \mathrm{~Hz}$.This research can make preparations for the follow-up study of the fault diagnosis of aluminum electrolysis.

\section{REFERENCES}

[1] Peng Yuhua, Wavelet transform and engineering application. Beijing: Tsinghua University press, 2005, pp. 88-97.

[2] Liu Mingcai, Wavelet analysis and its application, Beijing: Tsinghua University press, 2013, pp.22-46.

[3] Mallat S. A wavelet tour of signal processing. New York: A cademic Press, 1998, pp.46-50.

[4] Wang Huiqin, Wavelet analysis and application. Beijing: University of Posts and Telecommunications Press, 2011.

[5] Zhou Hao, "Aluminum electrolysis fault diagnosis technology based on Integrated Wavelet Neural Network,” Shenyang: Shenyang Construction University, 2011.

[6] Liu Yexiang, Li Zhe, Metallurgical Industry Press, Beijing, 2008, pp.145-157.

[7] P Maillard, M V Romerio. A stability criterion for an infinitely long Hall-Heroult cell [J]. Journal of computational and applied mathematies, 1996, pp.47-65.

[8] J. Anlille, M.Flueek, M V Romerio. Steady Velocity Field in Aluminlum Reduction cells Derived From Measurements of The andic current Flucuations. Light metals, 1994, pp. 305-31 